\title{
Collisionless driven reconnection in an open system
}

\author{
Ritoku Horiuchi ${ }^{1,2}$, Wenbing $\mathrm{Pei}^{2}$, and Tetsuya Sato ${ }^{1,2}$ \\ ${ }^{1}$ National Institute for Fusion Science, Toki 509-5292, Japan \\ ${ }^{2}$ The Graduate University for Advanced Studies, Toki 509-5292, Japan
}

(Received May 30, 2000; Revised January 29, 2001; Accepted March 5, 2001)

\begin{abstract}
Particle simulation studies of collisionless driven reconnection in an open system are presented. Collisionless reconnection evolves in two steps in accordance with the formation of two current layers, i.e., an ion current layer in the early ion phase and an electron current layer in the late electron phase. After the electron current layer is formed inside the ion current layer, the system relaxes gradually to a steady state when convergent plasma flow is driven by an external electric field with a narrow input window. On the other hand, when the convergent plasma flow is driven from the wide input window, magnetic reconnection takes place in an intermittent manner, due to the frequent formation of magnetic islands in the vicinity of neutral sheet.
\end{abstract}

\section{Introduction}

Collisionless magnetic reconnection plays an essential role in energetic active phenomena observed in the solar corona (Priest, 1982), the Earth's magnetosphere (Nishida, 1978) and the laboratory plasma (Ono et al., 1996; Yamada et al., 2000). Collisionless reconnection has been numerically studied based on two-fluid model (e.g. Biskamp et al., 1995), hybrid model (e.g. Shay et al., 1999), full particle model (e.g. Hesse and Winske, 1998). They have discussed the microscopic reconnection process such as reconnection rate, current sheet structure, and particle kinetic effects in the absence of an external driving source. The concept of an anomalous resistivity (Davidson and Gladd, 1975) which originates from the wave-particle interaction or the stochasticity of particle orbit (Horton and Tajima, 1990) has been introduced to explain collisionless reconnection. Recently three-dimensional full particle simulation (Zhu and Winglee, 1996; Horiuchi and Sato, 1999) has been carried out in order to clarify the relationship between an anomalous resistivity and collisionless reconnection.

In most of interesting reconnection phenomena the system is open and evolves dynamically with time. Therefore, microscopic kinetic process of collisionless reconnection is deeply connected with the dynamical evolution of a whole system. By carrying out two-dimensional particle simulation in the presence of an external driving source, Horiuchi and Sato $(1994,1997)$ have examined the kinetic process and have clarified that nonlinear evolution of collisionless reconnection is controlled not only by an internal microscopic process such as an electron dynamics or an ion dynamics in a current sheet, but also by an external global condition such as energy input rate or external driving flow. However, these analyses are restricted to the initial growing phase of collisionless driven reconnection because a peri-

Copy right (C) The Society of Geomagnetism and Earth, Planetary and Space Sciences (SGEPSS); The Seismological Society of Japan; The Volcanological Society of Japan; The Geodetic Society of Japan; The Japanese Society for Planetary Sciences. odic boundary condition is used at the downstream boundary.

Magnetic reconnection is often discussed with steady models. On the other hand, long time scale MHD simulations (Kitabata et al., 1996; Amo et al., 1995) have demonstrated that magnetic reconnection takes place intermittently when there exists a constant energy supply from the exterior region. Which situation is realized in collisionless reconnection? It is believed that steady reconnection is realized when the flux input rate into the system is balanced with the reconnection rate. However, this balance condition is not always assured because magnetic reconnection is controlled by two different processes with different time scales, i.e., an external global process and an internal microscopic process. In order to study a long time scale behavior of collisionless driven reconnection we developed a new open boundary model in which a free condition is used at the downstream boundary. By making use of both two-dimensional and three-dimensional kinetic open models, we will discuss the dynamics of collisionless driven reconnection in an open system that is subject to an external driving source.

The simulation model will be described in Section 2. Two types of open boundary models will be discussed in this paper: (a) semi-open model in which a periodic condition is used at the downstream boundary and plasma inflow exists at the upstream boundary, and (b) full open model in which a free condition is used at the downstream boundary and plasma inflow exists at the upstream boundary. The particle kinetic effect on collisionless driven reconnection is presented based on two-dimensional semi-open model in Section 3. Section 4 is devoted to the discussion on a long time scale behavior of collisionless driven reconnection based on two-dimensional full open model. In Section 5 we will discuss the relationship between an anomalous resistivity and collisionless reconnection by using three-dimensional semiopen model. 


\section{Simulation Model}

Three different types of electromagnetic particle codes are developed for the analyses of collisionless driven reconnection in two-dimensional and three-dimensional Cartesian coordinate systems (Horiuchi and Sato, 1994, 1997, 1999). As an initial condition we adopt a one-dimensional equilibrium with the Harris-type anti-parallel magnetic configuration as

$$
\begin{gathered}
\mathbf{B}(y)=\left(B_{x}(y), 0,0\right), \\
B_{x}(y)=B_{0} \tanh (y / L), \\
P(y)=B_{0}^{2} / 8 \pi \operatorname{sech}^{2}(y / L),
\end{gathered}
$$

where $B_{0}$ is a constant and $L$ is the scale height along the $y$-axis. There is a magnetically neutral sheet at $y=0$ in the initial equilibrium. The initial particle distribution is assumed to be a shifted Maxwellian with spatially constant temperature and average particle velocity, which is equal to the diamagnetic drift velocity.

The same input condition is used at the upstream boundary so that the plasma inflows are symmetrically driven from two input boundaries $\left(y= \pm y_{b}\right)$ by the external electric fields imposed in the $z$ direction. The distribution function of incoming particles at the input boundary is assumed to be a shifted Maxwellian with a constant temperature and the average velocity equal to the $\mathbf{E} \times \mathbf{B}$ drift velocity. The amplitude of driving field $E_{z d}(x, t)$ is set for zero at $t=0$, and increases with time while keeping a bell-shaped profile near the center and a flat profile in the periphery for an initial short moment. After then a constant profile is kept with maximum flux input rate $E_{0}$. Two types of boundary conditions are considered at the downstream boundary $\left(x= \pm x_{b}\right)$, i.e., (a) a periodic boundary condition, and (b) a free boundary condition. For the free boundary case field quantities are assumed that $E_{x}, E_{y}$ and $\partial E_{z} / \partial x$ are continuous at $x= \pm x_{b}$. The condition $\partial E_{z} / \partial x=$ finite allows the change in y-component of magnetic field which is the necessary condition for a magnetic island to go freely through the boundary. The boundary condition for particles is determined so as to keep the charge neutrality condition and the net number flux of particles associated with the fluid velocity in the vicinity of the downstream boundary. That is, there exist outgoing particles and incoming particles through the open boundary and their difference gives the net number flux. We can know the number flux of outgoing particles by observing them directly at the boundary. Under the assumptions that the net number flux of ions is the same as that of electrons, and the incoming particles satisfy the same distribution function as that just inside the open boundary, we can calculate the number fluxes of incoming ions and electrons, and get their velocities and positions. Thus, the total number of particles is a function of time in this open model.

\section{Two-Step Evolution of Collisionless Driven Re- connection}

In this section we review briefly the results of two-dimensional particle simulation for the semi-open model with the periodic downstream boundary (Horiuchi and Sato, 1994, 1997). Charged particles execute a complex thermal motion, which is called a meandering motion, in the vicinity of magnetically neutral sheet. The orbit amplitude of meandering motion is given by the geometrical mean of Larmor radius and the scale height of magnetic field. When there exists an external driving source, the current sheet evolves dynamically with time. That is, physical parameters, which control collisionless reconnection, becomes a function of time. In considering collisionless reconnection in the presence of an external driving source, it is important to examine the temporal evolution of physical parameters and their relationship.

Figures 1 and 2 show (1) the temporal evolution of an electric field along equilibrium current at the reconnection point ("reconnection electric field"), and (2) those of four spatial scales for the case of $m_{i} / m_{e}=50$ and $E_{0}=$ $-0.04 B_{0}$, where $t_{A}$ is the Alfven transit time, $d_{h}, d_{j z}, l_{m i}$,

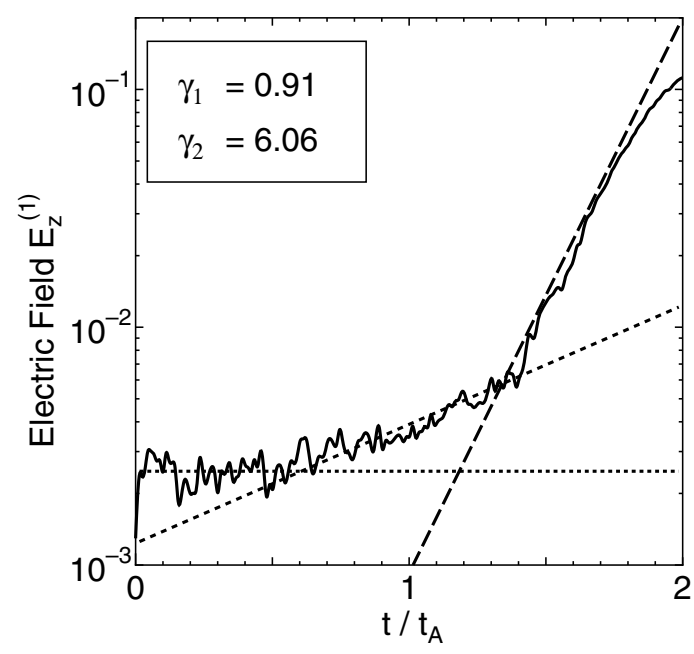

Fig. 1. Temporal evolution of reconnection electric field for the case of periodic downstream boundary.

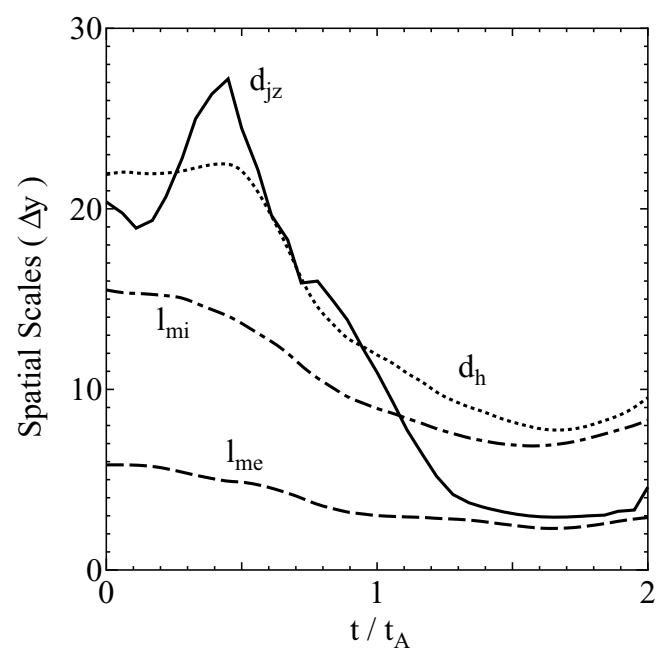

Fig. 2. Temporal evolutions of four spatial scales for the case of periodic downstream boundary. 
and $l_{m e}$ are the half-width of the mass density profile, that of the current density profile, the average amplitude of ion meandering orbit, and that of electron meandering orbit, respectively. Figure 1 indicates that collisionless reconnection takes place in two steps, i.e., the slow reconnection in the early phase $\left(0.6 t_{A}<t<1.3 t_{A}\right)$ and the fast reconnection in the late phase $\left(1.3 t_{A}<t<1.8 t_{A}\right)$. The electric field begins to grow slowly as soon as both $d_{h}$ and $d_{j z}$ start decreasing as a result of the compression by the convergent plasma flow in the slow reconnection phase. The width of the current layer is almost the same or a little larger than the ion thermal scale $l_{m i}$ in this phase.

Because most of the ions are unmagnetized in the current layer ("ion current layer") while the electrons are magnetized, the input flow (Poynting flux) no longer works on thinning the ion current layer, but it continues to compress the electron profile. Thus, electron current layer is created inside the ion current layer due to the finite ion Larmor radius effect. When the fast reconnection phase sets in, the inclination of the growth curve steepens suddenly and the half-width of electron current layer approaches the electron thermal scale $l_{m e}$. These results lead us to the conclusion that the slow reconnection is triggered by the ion meandering motion effect while the fast reconnection is triggered by the electron meandering motion effect.

From the simulation runs with a various values of ion mass, electron mass, and the driving electric field (Horiuchi and Sato, 1994, 1997), it is found that the fundamental properties of collisionless driven reconnection are determined by two physical processes, i.e., the external process which controls the flux input rate into the current layer, and the internal process which controls the particle kinetic mechanism leading to collisionless driven reconnection in the current layer, and they are not independent processes but deeply connected to each other.

\section{Dynamics of Collisionless Reconnection in a Long Time Scale}

In this section we discuss the particle simulation in a long time scale based on the two-dimensional full open model with the free downstream boundary. The simulation is carried out on a $512 \times 128$ point grid by making use of 6.4 million particles. The main parameters are in the followings: $m_{i} / m_{e}=25, \omega_{p e} / \omega_{c e}=3.5, \omega_{c e} \Delta t=0.02, L=$ $0.8 y_{b} \approx 3 \rho_{i}$, where $\rho_{i}$ is the ion Larmor radius, and $\omega_{c e}$ is defined by using a constant field $B_{0}$ in the initial equilibrium. Figure 3 shows the spatial profiles of the driving electric field imposed at the input boundary at five different time periods, i.e., $t \omega_{c e}=0,10.2,20.4,81.6$, and 143. Here, the spatial size of initial bell-shaped profile is $x_{d}=0.84 x_{b}$, and the amplitude of the uniform profile in the late phase is $E_{0}=-0.04 B_{0}$. We call the parameter $x_{d}$ input window size, because the input velocity is locally enhanced within this region. In order to examine the dependence of the reconnection process on the profile of driving electric field, we performed simulation runs with different values of $E_{0}$ and $x_{d}$ and found that the long time scale behavior is insensitive to the value of $E_{0}$ and depends strongly on the value of the window size $x_{d}$. In the following, we discuss the simulation results for the case of $E_{0}=-0.04 B_{0}$.

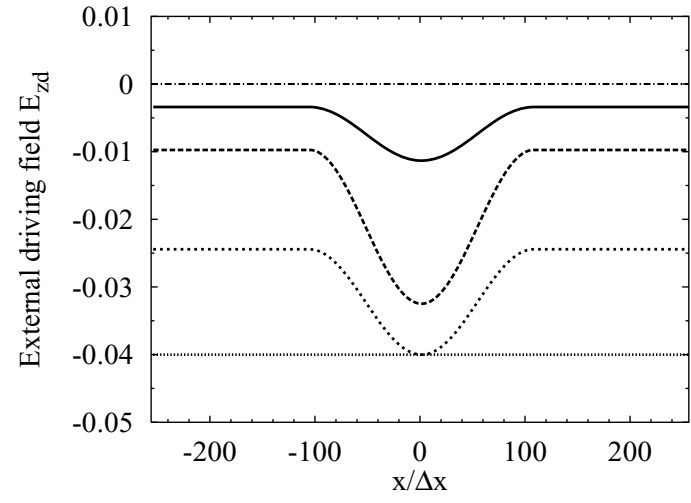

Fig. 3. Spatial profiles of driving electric field at five different time periods for the parameters $x_{d}=0.84 x_{b}$ and $E_{0}=-0.04 B_{0}$.

Time $=0$

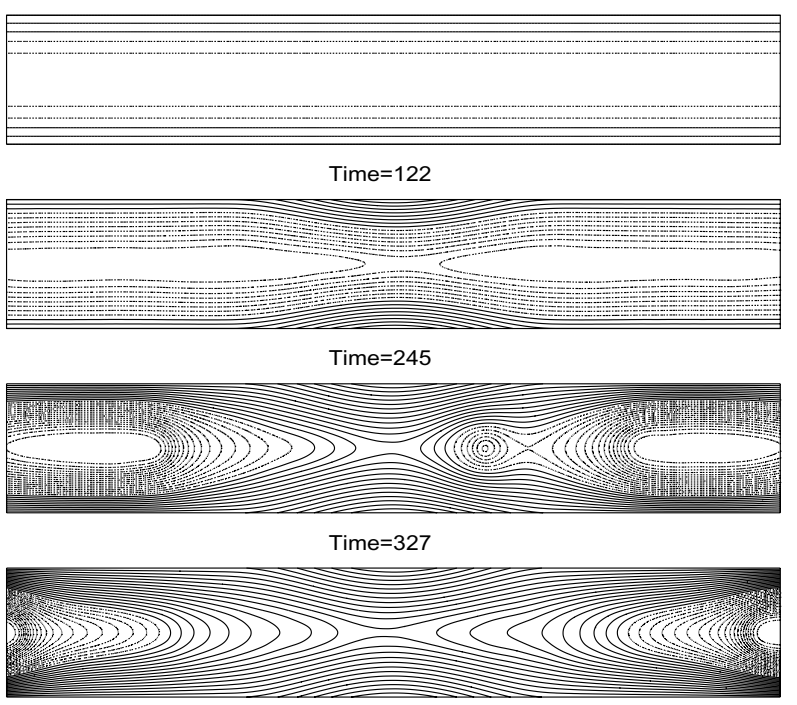

Time $=490$

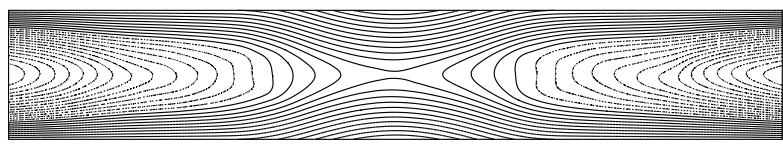

Time $=735$

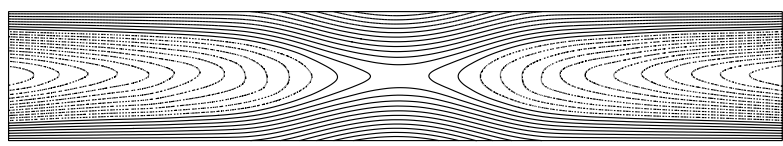

Fig. 4. Temporal evolution of magnetic field for the narrow window case of $x_{d} / 2 x_{b}=0.42$.

First, let us consider the case of narrow input window $\left(x_{d} / 2 x_{b}=0.42\right.$ or $\left.x_{d}=18 \rho_{i}\right)$. Figure 4 shows the temporal evolution of the magnetic field. The convergent flow supplied from the top and bottom boundaries compresses the current sheet. Magnetic reconnection sets in at the center of the simulation domain at $t \omega_{c e} \approx 122$ (second panel). A small island appears near the reconnection point and grows with time (third panel). After some periods this island disappears due to the merging with right bulk plasma. Any magnetic island is not created ever after and so global field topology remains unchanged. That is, the system reaches a 


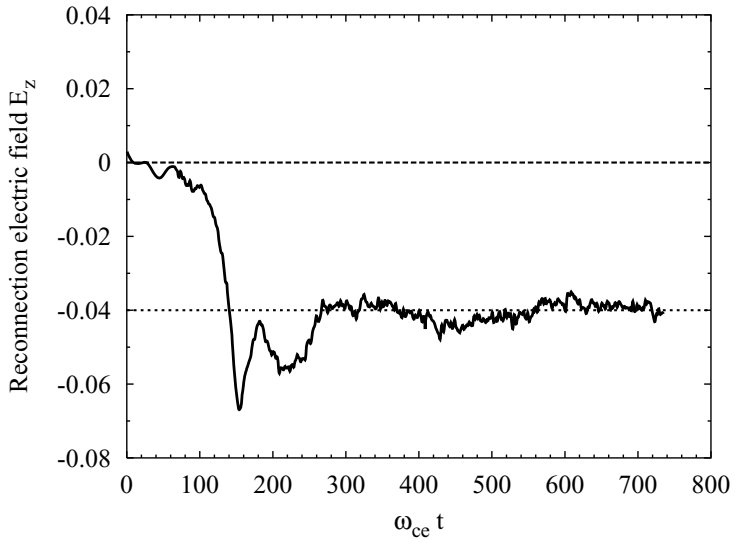

Fig. 5. Temporal evolutions of reconnection electric field for the narrow window case of $x_{d} / 2 x_{b}=0.42$.

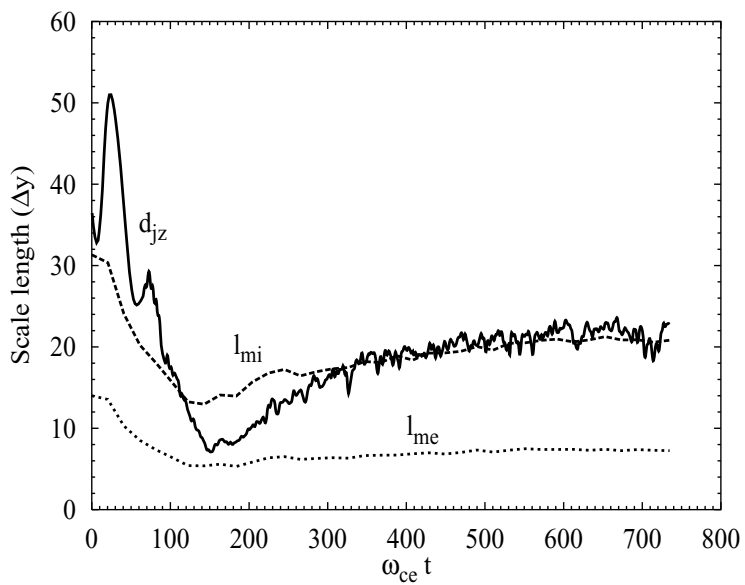

Fig. 6. Temporal evolutions of three spatial scales for the narrow window case of $x_{d} / 2 x_{b}=0.42$.

steady reconnection state.

Figure 5 plots the time history of an electric field at the reconnection point for the same case as Fig. 4 where the dotted line stands for the value of driving electric field. The behavior of the reconnection field for an initial growing phase is very similar to the simulation results discussed in Section 3. After over-shooting the field approaches the external driving field. The reconnection field tends to fluctuate around the value of the external field with a small amplitude for $t \omega_{c e}>300$. Figure 6 is the time history of three spatial scales. The width of current layer approaches the electron meandering scale $l_{m e}$ for an initial growing phase in accordance with the formation of the electron current layer. However, it tends to increase for $t \omega_{c e}>160$ and is kept nearly equal to the ion meandering scale $l_{m i}$ in a steady state. The electron current density is described by the superposition of an electron density profile and an average electron velocity profile. The electron density profile has an ion scale because it takes almost the same form as the ion density profile. On the other hand, the electron velocity profile, which has an electron scale, is strongly dependent on the acceleration mechanism in the electron current layer. If a locally enhanced profile of average electron velocity is formed, the electron current layer is clearly visible inside the ion current layer. Figure 6 suggests that the localized peaked profile of electron velocity formed in the initial growing phase is damped down and the electron current layer becomes invisible in the steady state. The mechanism controlling the current sheet structure is discussed in detail by Pei et al. (2001). This result is in good agreement with the experimental one of the MRX (Magnetic Reconnection Experiment) (Yamada et al., 2000).

Now, let us discuss the simulation results for the wide window case $\left(x_{d} / 2 x_{b}=0.83\right.$ or $\left.x_{d}=36 \rho_{i}\right)$. Figure 7 il-

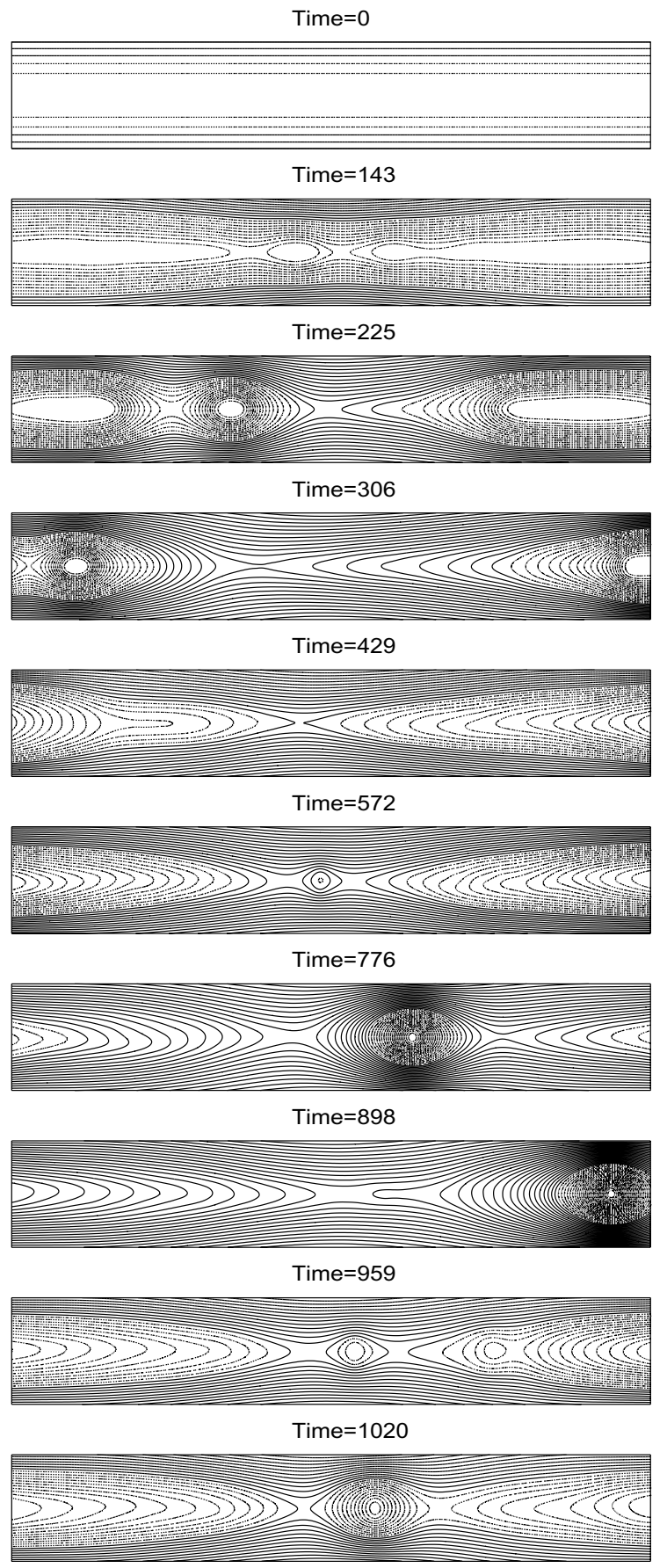

Fig. 7. Temporal evolutions of magnetic field for the wide window case of $x_{d} / 2 x_{b}=0.83$. 
lustrates the spatial profile of magnetic field at ten different time periods. A small island is created at the center of current sheet at $t \omega_{c e}=143$. This island grows with time while moving to the left. The system relaxed to a state without any island after this island moves out through the left boundary $\left(t \omega_{c e}=429\right)$. After a short period a small island is created again at the center at $t \omega_{c e}=572$. This island moves to the right while increasing its size and disappears through the right boundary. This process is repeated again and again. An elongated current sheet along the $x$-axis is created as a result of the plasma compression over a relatively long range for the wide window case. The length of the current sheet is roughly estimated as $L_{c s} \approx 10 \rho_{i}$. This means that the current sheet becomes unstable against a collisionless tearing instability. Consequently, the system cannot reach any steady state due to the frequent formation of magnetic islands in the current sheet. Figure 8 plots the temporal evolution of the reconnection electric field for the same case as Fig. 7. One can see in Fig. 8 that, compared with the narrow window case, the reconnection field is fluctuating around the value of the external field with a relatively larger amplitude during the simulation, and never reaches a constant value. It

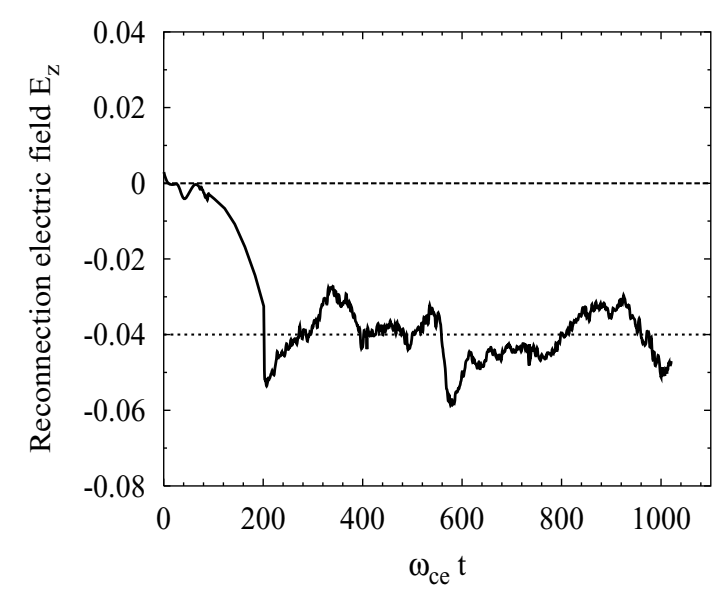

Fig. 8. Temporal evolutions of reconnection electric field for the wide window case of $x_{d} / 2 x_{b}=0.83$.

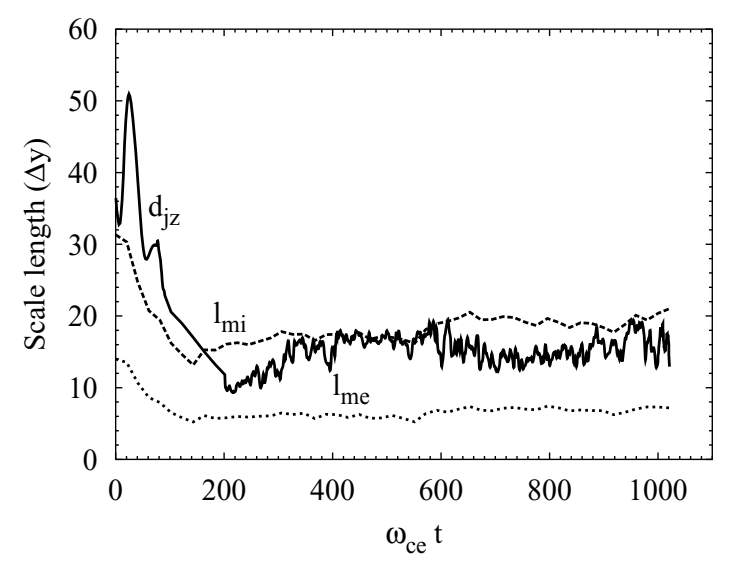

Fig. 9. Temporal evolutions of three spatial scales for the wide window case of $x_{d} / 2 x_{b}=0.83$. is concluded that, for wide window case, collisionless reconnection takes place intermittently, but not in a steady way.

We can see another interesting phenomenon of an intermittent reconnection in Fig. 9. The width of the current layer $d_{j z}$ changes between the ion scale $l_{m i}$ and the electron scale $l_{m e}$ in accordance with the fluctuation of the reconnection electric field. When the reconnection field exceeds the driving field, the width decreases below $l_{m i}$ and the electron current layer becomes visible inside the ion current layer. It is interesting to note that this period corresponds to the growing phase of magnetic islands in the neutral sheet. On the other hand, when the reconnection field has a value close to the driving field, the width returns to $l_{m i}$ and the electron current layer becomes invisible. In this way, collisionless reconnection takes place intermittently while changing the spatial structure of the current sheet dynamically for wide window case.

\section{Collisionless Driven Reconnection in Three Di- mensions}

Winske (1981), Tanaka and Sato (1981), and Ozaki et al. (1996) have found from two-dimensional particle simulation that two kinds of plasma instabilities grow in the current layer, i.e., the lower hybrid drift instability (LHDI) (Krall and Liewer, 1971) and the drift kink instability (DKI) (Zhu and Winglee, 1996; Daughton, 1999). The LHDI grows in the periphery of the current layer, but it cannot penetrate into a high beta region in the vicinity of the neutral sheet. Ozaki et al. (1996) have pointed out that the DKI is excited near the neutral sheet after the saturation of the LHDI and can be a cause of anomalous resistivity at the neutral sheet. Linear analysis (Daughton, 1999) has revealed that the growth rate of the DKI has a peak around $k_{z} L \approx 1.0$ but it decreases with the mass ratio $m_{i} / m_{e}$ for a fixed $\rho_{i} / L$, where $k_{z}$ is a wave number along an equilibrium current. Thus, the growth rate for the real mass ratio and $\rho_{i} / L=1.0$ is too small to explain substorm onset even for $k_{z} L \approx 1.0$. Another important feature is that the DKI is driven by the velocity difference between ions and electrons and therefore its growth strongly depends on the ratio of ion Larmor radius to the current sheet scale $\rho_{i} / L$ in the Harris equilibrium (Daughton, 1999). Let us examine the dependence of the growth rate on the ratio $\rho_{i} / L$ by changing the mass ratio. Figure 10 shows the result of two-dimensional particle simulation without any background population where the growth rate is plotted as a function of $\rho_{i} / L$ for three different ion masses and $k_{z} L=0.8$. It is clear from Fig. 10 that the growth rate increases with the ratio $\rho_{i} / L$. It has a remarkably large value even for a large mass ratio if $\rho_{i} / L>1$.

In a real system there are many causes that change the current sheet structure. The external plasma inflow is a typical example of such causes. In many cases, the current sheet width becomes a function of time under the influence of plasma inflow and outflow, as was seen in the previous sections. Three-dimensional treatment is needed to examine the generation of an anomalous resistivity in the dynamically evolving open system with plasma inflow and outflow. Figure 11 shows the result of the three-dimensional particle simulation in the presence of an external driving source where the Fourier amplitude of $n=1$ mode of $B_{x}$ at the 


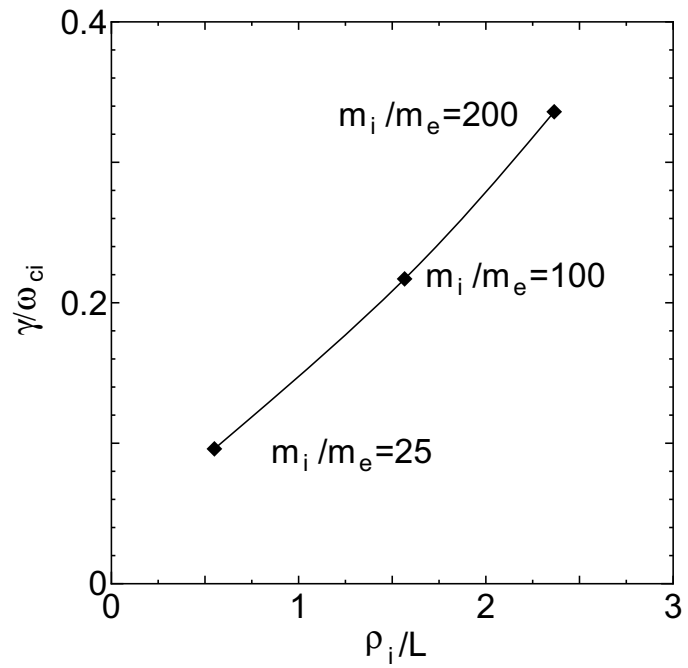

Fig. 10. Growth rate of the drift kink mode as a function of $\rho_{i} / L$ for three different ion masses.

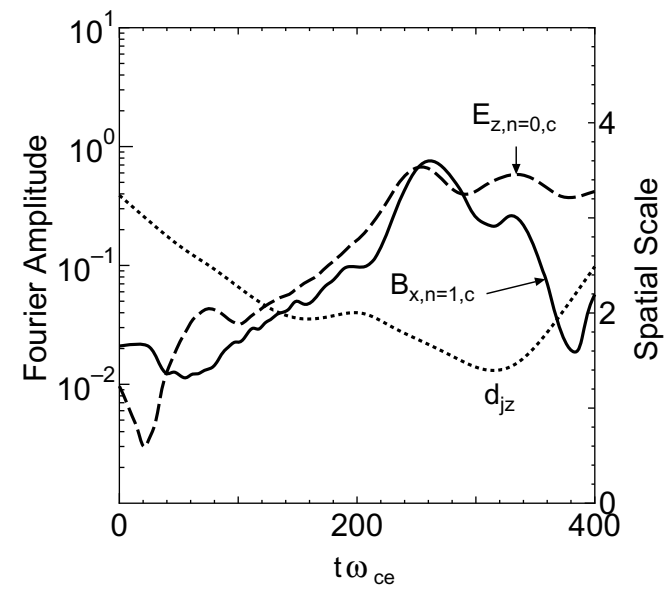

Fig. 11. Temporal evolutions of the Fourier modes and the half-width of current layer for the three-dimensional simulation where the solid, dashed, and dotted lines stand for the drift kink mode, the reconnection electric field, and the half-width, respectively.

center of current sheet (solid), the reconnection electric field (dashed), and the half-width of the current layer (dotted) are plotted for the parameters $m_{i} / m_{e}=100, \rho_{i} / L=1.2$, $k_{z} L \approx 2$, and $E_{0}=-0.02 B_{0}$. From the simulation parameters we expect that the growth rate of the DKI for the initial profile is too small to detect its growth during the simulation period. The Poynting flux, which is supplied into the simulation domain from the exterior region, moves towards the current layer together with the convergent plasma flow, while compressing the current profile. The compression by the convergent plasma flow increases the growth rate of the DKI. One can see the kink-like deformation of current sheet in the driven case as well as in the non-driven case. It is likely that this deformation is caused by the excitation of the DKI, although the characteristic features of this mode are slightly different from the DKI in the non-driven case. For example, the growth rate of this mode is estimated to be $\gamma / \omega_{c i} \approx 1.5$ from the Fourier amplitude of $B_{x}$ during $90<\omega_{c e} t<190$ when $\rho_{i} / L=1.5$. This value is several times as large as that in the non-driven case (Daughton, 1999). The reason may be partly due to the fact that the magnetic field is magnified twice through the compression by the convergent plasma flow, and the ion drift velocity along the equilibrium current is fairly large $\left(k_{z} v_{d i} \approx 6 \omega_{c i}\right.$ for $n=1)$. Because most of ions inside the current layer are unmagnetized, the electric field or the Poynting flux, which is carried by the convergent plasma motion, penetrates into the current layer due to the particle kinetic effect (Horiuchi and Sato, 1994, 1997). When the Poynting flux reaches the neutral sheet, collisionless reconnection is triggered by the convective electric field.

Once magnetic reconnection takes place, a generated fast plasma flow carries away the plasma near the reconnection point towards the downstream. One can see in Fig. 11 that, after the reconnection electric field reaches its saturation level, the amplitude of the DKI decreases and the width of the current layer increases. These phenomena indicate that the reconnection flow carries away the plasmas faster than the flux is accumulated at the reconnection point by the input flow. Consequently, the current profile is flattened in the vicinity of the reconnection point and the DKI becomes stable there. Figure 12 shows the perspective views of the current sheet at $t \omega_{c e}=408$ in the real space for the same case as Fig. 11. Reconnection point appears almost along the straight midline $(x=0)$ in the current sheet (see a green color in the central region). We cannot see any modification of the current sheet by the DKI in the vicinity of reconnection point, although there appears the $n=1$ modification of the current sheet along the $z$-axis in the downstream region. The simulation is terminated at $t \omega_{c e}=408$ because the reconnection plasma flow approaches the downstream boundary at which the periodic condition is adopted. The real frequency of the kink mode is observed as $\omega_{r} \approx 4 \omega_{c i}$, and thus the simulation time corresponds to only three oscillation periods of the drift kink mode. This time is too short to judge whether the DKI works as an anomalous resistivity leading to collisionless reconnection in an open system.

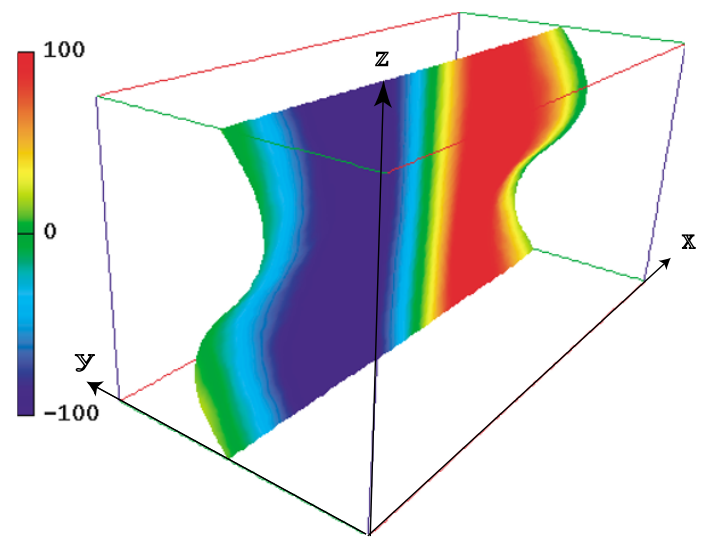

Fig. 12. Perspective views of the current sheet at $t \omega_{c e}=408$ for the same case as Fig. 11 where the current sheet is defined by the condition $B_{x}=0$, red and blue colors stand for the positive and negative values of magnetic field $B_{y}$. 
However, it is not likely that the DKI is a primary cause of collisionless reconnection in the presence of an external driving source.

\section{Summary}

Based on two-dimensional and three-dimensional kinetic models, we have investigated the dynamics of collisionless driven reconnection in an open system that is subject to an external driving source.

It is found from two-dimensional kinetic simulations with the periodic downstream boundary that collisionless reconnection evolves in two steps in accordance with the formation of two current layers, i.e., an ion current layer in the early phase and an electron current layer in the late phase. The slow reconnection in the early phase is triggered by the ion meandering motion effect while the fast reconnection in the late phase is triggered by the electron meandering motion effect.

A long time scale behavior of collisionless reconnection is investigated by using two-dimensional open model with the free downstream boundary. When the convergent plasma flow is driven by an external electric field with a narrow input window $\left(x_{d}=18 \rho_{i}\right)$, the system relaxes to a steady state in which the reconnection rate is determined by the external electric field. On the other hand, when the input window is wide $\left(x_{d}=36 \rho_{i}\right)$, magnetic reconnection takes place in an intermittent manner due to the frequent formation of magnetic islands in the neutral sheet.

The relationship between the anomalous resistivity associated with wave-particle interaction and collisionless reconnection has been investigated by means of three-dimensional particle simulation in the presence of an external driving source. It is found that the drift kink instability, which is excited through the compression of current sheet by the divergent plasma flow in the early phase, is stabilized by the reconnection flow. Thus, it is likely that a primary cause of collisionless reconnection is not the drift kink instability, but the particle kinetic effect in the presence of an external driving source. However, full understanding of the drift kink instability in the presence of an external driving source needs the analysis in a much longer time scale with a larger simulation box in a three-dimensional open system. This problem will be solved in a near future.

\section{References}

Amo, H., T. Sato, A. Kageyama, K. Watanabe, R. Horiuchi, T. Hayashi, Y. Todo, T. H. Watanabe, and H. Takamaru, Intermittent energy bursts and recurrent topological change of a twisting magnetic flux tube, Phys. Rev., E51, 3838-3841, 1995.

Biskamp, D., E. Schwarz, and J. F. Drake, Ion-controlled collisionless magnetic reconnection, Phys. Rev. Lett., 75, 3850-3853, 1995.

Daughton, W., The unstable eigenmodes of a neutral sheet, Phys. Plasmas, 6, 1329-1343, 1999.

Davidson, R. C. and N. T. Gladd, Anomalous transport properties associated with the lower-hybrid-drift instability, Phys. Fluids, 18, 1327, 1975.

Hesse, M. and D. Winske, Electron dissipation in collisionless magnetic reconnection, J. Geophys. Res., 103, 26479-26486, 1998.

Horiuchi, R. and T. Sato, Particle simulation study of driven magnetic reconnection in a collisionless plasma, Phys. Plasmas, 1, 3587-3597, 1994.

Horiuchi, R. and T. Sato, Particle simulation study of collisionless driven reconnection in a sheared magnetic field, Phys. Plasmas, 4, 277-289, 1997.

Horiuchi, R. and T. Sato, Three-dimensional particle simulation of plasma instability and collisionless reconnection in a current sheet, Phys. Plasmas, 6, 4565-4574, 1999.

Horton, W. and T. Tajima, Decay of correlations and the collisionless conductivity in the geomagnetic tail, Geophys. Res. Lett., 17, 123-126, 1990.

Kitabata, H., T. Hayashi, T. Sato, R. Horiuchi, K. Watanabe, A. Kageyama, T. H. Watanabe, Y. Todo, and H. Takamaru, Impulsive nature in magnetohydrodynamic driven reconnection, J. Phys. Soc. Japan, 65, 3208 3214, 1996

Krall, N. A. and P. C. Liewer, Low-frequency instability in magnetic pulses, Phys. Rev., 4, 2094-2103, 1971.

Nishida, A., Geomagnetic Diagnostics of the Magnetoshpere, p. 38, Springer-Verlang, New York, 1978.

Ono, Y., M. Yamada, T. Akao, T. Tajima, and R. Matsumoto, Ion acceleration and direct ion heating in three-component magnetic reconnection, Phys. Rev. Lett., 76, 3328-3331, 1996.

Ozaki, M., T. Sato, R. Horiuchi, and the Complex Simulation Group, Electromagnetic instability and anomalous resistivity in a magnetic neutral sheet, Phys. Plasmas, 3, 2265-2274, 1996.

Pei, W., R. Horiuchi, and T. Sato, Long time scale evolution of collisionless driven reconnection in a two-dimensional open system, Phys. Plasmas, 2001 (in press).

Priest, E. R., Solar Magnetohydrodynamics, Reidel, Dordrecht, 1982.

Shay, M. A., J. F. Drake, B. N. Rogers, and R. E. Denton, The scaling of collisionless, magnetic reconnection for large systems, Geophys. Res. Lett., 26, 2163-2166, 1999.

Tanaka, M. and T. Sato, Simulations on lower hybrid drift instability and anomalous resistivity in the magnetic neutral sheet, J. Geophys. Res., 86, 5541-5552, 1981.

Winske, D., Current-driven microinstabilities in a neutral sheet, Phys. Fluids, 24, 1069-1076, 1981.

Yamada, M., H. Ji, S. Hsu, T. Carter, and R. Kulsrud, Experimental investigation of the neutral sheet profile during magnetic reconnection, Phys. Plasmas, 7, 1781-1787, 2000.

Zhu, Z. and R. M. Winglee, Tearing instability, flux ropes, and the kinetic current sheet kink instability in the Earth's magnetotail: A threedimensional perspective from particle simulations, J. Geophys. Res., 101, 4885-4897, 1996.

R. Horiuchi (e-mail: hori@tcsc.nifs.ac.jp), W. Pei, and T. Sato 